\title{
A NEW ARGENTINE GENUS OF THERIINI (HYMENOPTERA, ICHNEUMONIDAE)*
}

\author{
By Charles C. Porter \\ Department of Biological Sciences, Fordham University, \\ Bronx, New York 10458
}

Recent fieldwork in subtropical humid forests of Salta and Corrientes Provinces of north Argentina has revealed a new genus of the anomalonine ichneumonid tribe Theriini (as defined by Townes, 1971, p. 155) and therein most closely related to the Palaearctic and Indo-Papuan genus Heteropelma Wesmael.

\section{Stangepelma, new genus}

$$
\text { (Figures 1-5, Map 1) }
$$

Clypeus: median $2 / 3$ of apical margin practically truncate. Mandible: lower tooth about $1 / 3$ as long as upper. Occipital carina: joins base of mandible. Frons: with a high vertical lamella between antennal sockets. Mesoscutum: without a transverse suture before scuto-scutellar groove. Scutellum: in dorsal view gently convex laterad and weakly concave on median $1 / 3$; in profile weakly convex. Postpectal carina: complete. Propodeum: of normal shape, gaster attached only a little above hind coxae; pleural carina absent so that propodeum is not distinctly separated from metapleuron. Fore coxa: without a transverse carina on ventral surface. Mid tibia: with one apical spur. Hind femur: clavate, its basal half very long and slender, 0.04 as deep as length of femur, and its apical half abruptly swollen, $0.11-0.12$ as deep as length of femur. Hind tarsus: basitarsus $1.8-1.9$ as long as segments 2-5 of hind tarsus; second segment in male with a weak depression below just beyond middle. Tarsal claws: not pectinate, those of front and mid tarsus with an approximately 90 degree bend just beyond middle, those of hind tarsus with a 100 degree bend at middle and a lobe at base. Wing venation: intercubitus joins cubitus basad of second recurrent vein by about 1.0 (male)-

*Manuscript received by the editor October 14, 1976 
1.5 (female) the length of intercubitus; nervellus intercepted between its upper 0.33 and 0.40 ; metacarpella with a series of 6-7 widely spaced hamuli.

TYPE SPECIES: Stangepelma argentinum, new species.

Discussion: Within its tribe Stangepelma shows special affinity to Heteropelma Wesmael and Tanypelma Townes, with which it shares such characters as a high lamella between the antennal sockets, a complete postpectal carina, and sharply bent hind tarsal claws. From the foregoing genera, it differs in its single mid tibial spur, basally very slender and apically inflated hind femur, second male hind tarsal segment with a weak depression below (Tanypelma has no depression, Heteropelma a flattened and impressed area), and by having a distal series of 6-7 widely spaced hamuli (12-18 closely spaced hamuli in Heteropelma and Tanypelma). The single mid tibial spur is a feature shared among Theriini only by the eastern Palaearctic Brachynervus Uchida $^{1}$ and Brachynervus also resembles Stangepelma because it has only 7 widely spaced metacarpellan hamuli. However, Stangepelma and Brachynervus do not seem closely related, since Brachynervus has many features which set it off from all other theriines (mesoscutum with a transverse suture before scutoscutellar groove, postpectal carina absent, occipital carina joining hypostomal carina instead of mandibular base, intercubitus far basad of second recurrent vein, and propodeum globose with its apex raised away from the hind coxae so that the gastric socket is distant from the hind coxae). Apart from the features already mentioned, Stangepelma agrees almost perfectly with Townes' (1971, p. 158) description of the Palaearctic and Indo-Papuan Heteropelma but shows the following additional differences from the eastern Nearctic Tanypelma: apex of clypeus truncate instead of weakly convex, lower tooth of mandible 0.3 as long as upper instead of only a little shorter than upper, scutellum gently instead of strongly convex, front and middle tarsal claws with a 90 degree bend instead of with a moderately strong curve, and nervellus intercepted definitely above instead of near the middle.

\footnotetext{
'According to Gauld (1976, p. 28), the Malayasian Heteropelma perornatum Cameron "sometimes has the inner tibial spur very reduced and although it appears to have a single spur on the mid tibia, two are in fact present."
} 

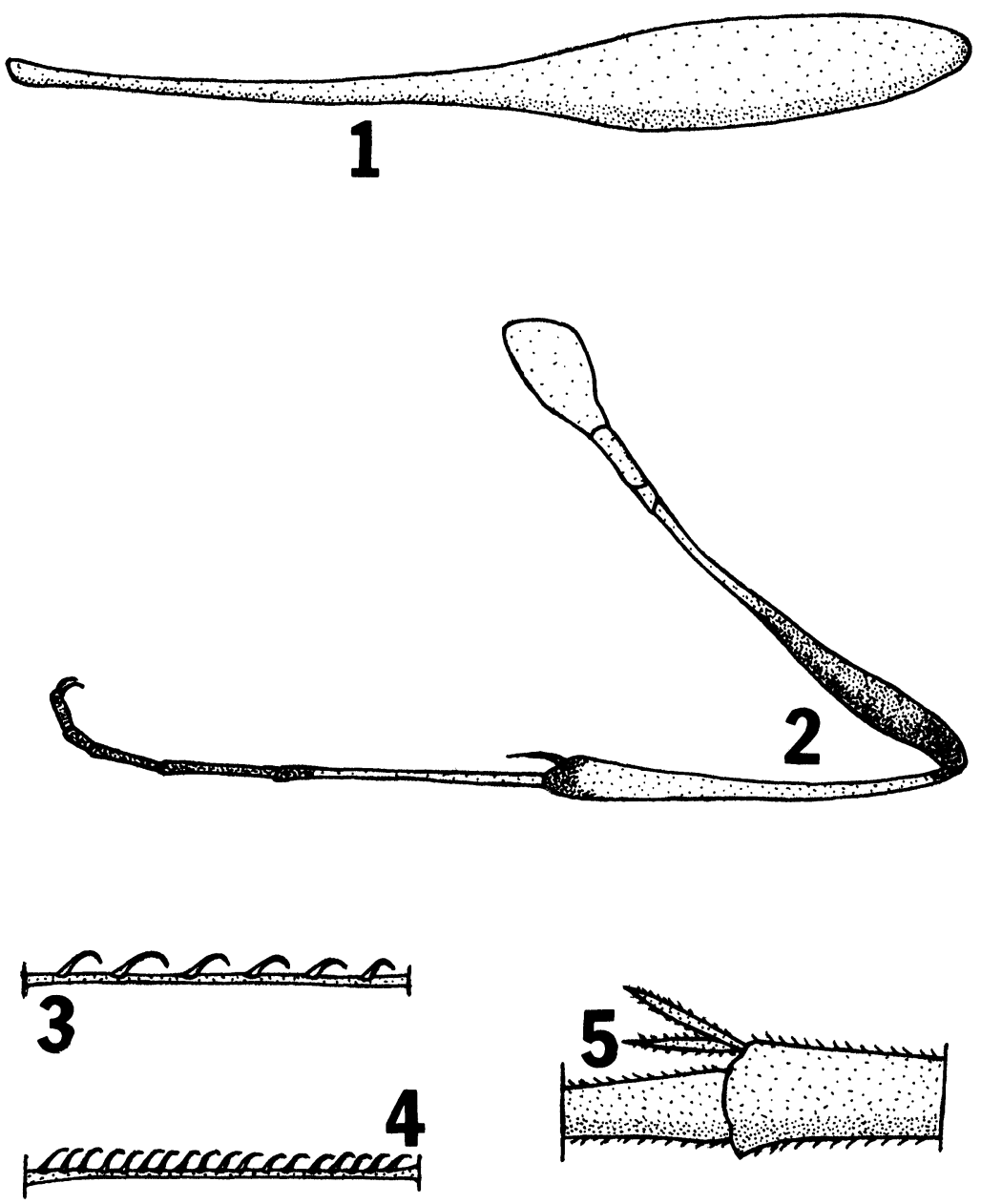

Fig. 1. Stangepelma argentinum $\mathrm{n}$. sp., female holotype. Lateral view of hind femur. Fig. 2. Stangepelma argentinum n. sp., female holotype. Anterior view of left mid leg, showing color pattern and single mid tibial spur. Fig. 3. Stangepelma argentinum $\mathrm{n}$. sp., female holotype. Metacarpellan hamuli. Fig. 4. Tanypelma fulvicorne Townes, female. Metacarpellan hamuli. Fig. 5. Tanypelma fulvicorne Townes, female. Apex of mid tibia and base of mid basitarsus, showing the two mid tibial spurs. 
Therion Curtis, with several undescribed Argentine species, is the only other genus of Theriini currently known from the Neotropics. It differs from Stangepelma in having two mid tibial spurs, an incomplete postpectal carina, and hind tarsal claws curved at less than 90 degrees.

Generic name: For Dr. Lionel A. Stange of the Instituto Miguel Lillo, who collected the holotype of Stangepelma argentinum.

Stangepelma argentinum, new species

Holotype: female, ARGENTINA (Salta: $1 \mathrm{~km}$. Oe. Aguas Blancas, 11 August 1976, L. A. Strange, C. C. Porter). (Tucumán). Paratype: male, ARGENTINA (Corrientes: Las Marías, ca. Gdor. Virasoro, 7 November 1971, C. Porter). (Porter).

Female: Color: antenna black with a yellow blotch on scape below; head and mesosoma shining black with yellow on basal $2 / 3$ of mandible, labrum, clypeus, face, frontal orbit to slightly above antennal socket, for a short distance on vertical orbit, on most of temporal orbit (becoming very narrow above), broadly on lateral swellings of scutellum, briefly on base on tegula, and on most of subalarum; first gastric segment shining piceous black; second segment shining piceous black on tergite and brownish yellow on sternite; and succeeding segments shining pale brown, becoming more or less dusky dorsad and more yellowish ventrad; fore leg pale yellow with considerable light brown on apical $2 / 3$ of femur, especially below, a little brown on base of tibia, brown on much of third tarsomere, and dark brown on tarsomeres 4-5; mid leg pale yellow with dark brown, becoming more yellowish anteriad, on about apical $2 / 5$ of femur, a little brown on base and apex of tibia, and dark brown on tarsomeres 2-5 and tip of 1; hind leg with coxa shining black above and yellow below; trochanter, trochantellus, and femur shining black with yellow below on extreme base of trochanter, yellow grading apically into brownish below on trochantellus, a little piceous below on basal half of femur, and tibia and tarsus dull black with yellow on sub-basal $1 / 5$ of tibia below; wings hyaline with a slight brownish tinge and the stigma dark brown except for a small whitish area at base.

Length of fore wing: $8.1 \mathrm{~mm}$. Flagellum: elongate and practically filiform, first segment 5.0 as long as deep at apex, second segment 2.1 as long as deep at apex. Face: 1.4 as high as wide at 
anterior tentorial pits, 1.8 as wide at level of antennal sockets as at anterior tentorial pits; with large, strong, mostly adjacent or subadjacent to a little sparser punctures. Clypeus: on each side subapically with a broad, weakly defined swelling. Temple: moderately receding behind eye; at its upper $1 / 4$ about 0.56 as long as eye in lateral view. Pronotum: with a broad and foveolate dorsal submarginal groove; its lateral surface smooth and polished for some distance behind epomia but with coarse longitudinal wrinkles toward hind margin. Mesoscutum: notauli strong, apically confluent, reaching about $9 / 10$ the length of mesoscutum; surface throughout with strong, subadjacent to a little sparser punctures. Mesopleuron: on most of its dorsal 1/3 with strong longitudinal wrinkling but on ventral $2 / 3$ generally smooth and polished with numerous well spaced small punctures that become progressively denser rearward. Metapleuron: with strong reticulate wrinkling like that of propodeum; submetapleural carina low, defined only on about basal 2/3. First gastric tergite: 8.2 as long as wide at apex. Ovipositor: sheathed portion 0.6 as long as apical depth of gaster.

MALE: differs from female as follows: Color: scape entirely black; no yellow on frontal orbit; yellow on temporal orbit limited to a short line near mid-height of eye; scutellum entirely black; tegula entirely black; second gastric segment almost entirely black; and succeeding gastric segments darker than in female, blackish dorsad and becoming dull yellowish brown ventrad; front femur rather dark brown below and posterio-dorsally on apical 3/4 and with yellow staining longitudinally between the dark zones; fore tarsus with weak brown staining on fourth segment and dark brown only on fifth; mid femur uniformly black on a little more than apical half; mid tibia dark brown on basal $1 / 8$ and again on apical 1/3; about apical $1 / 3$ of mid basitarsus dark brown; hind femur with dull yellow to piceous below on basal $1 / 2$, which is brightest close to base and again on a fairly broad, dorsally prolonged area just before mid length.

Length of fore wing: $7.4 \mathrm{~mm}$. Flagellum: first segment 4.7 as long as deep at apex, second segment 2.3 as long as deep at apex. Face: 1.6 as high as wide at anterior tentorial pits, 1.4 as wide at level of antennal sockets as at anterior tentorial pits. Clypeus: subapical lateral swellings more strongly defined than in female and occupying all but median $1 / 4$, which is depressed between 


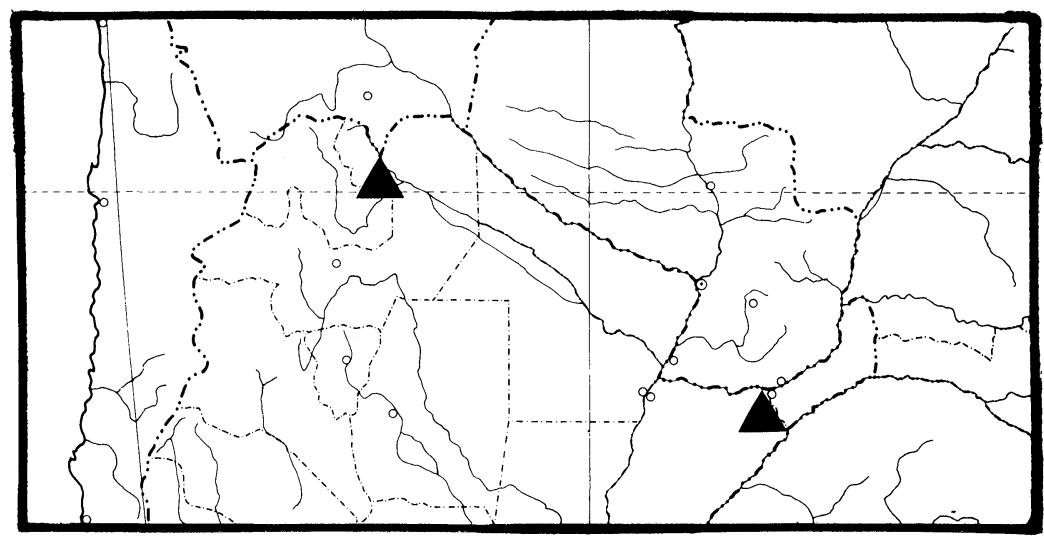

Map 1. Northern Argentina, showing distribution of Stangepelma argentinum.

them. Temple: at its upper $1 / 4$ only about 0.36 as long as eye in lateral view and more strongly receding behind eye than in female. Mesoscutum: notauli reaching about 8/10 the length of mesoscutum; punctures even sharper and denser than in female, largely adjacent. First gastric tergite: 8.7 as long as wide at apex.

TyPES: The female holotype will be deposited at Tucumán, Argentina in the collection of the Instituto Miguel Lillo of the Universidad Nacional de Tucumán. The male paratype will be retained in the collection of Charles C. Porter at 301 North 39th Street, McAllen, Texas.

FIELD Notes: The female holotype was netted among roadside weeds in bright sun. The type locality is in Selva Chaqueña, a subtropical deciduous forest which in northeastern Salta and Jujuy Provinces of Argentina forms a transition between the lowland Dry Chaco scrub to the east and the mostly evergreen Selva Tucumano-Boliviana at the base of the Andes to the west.

The male paratype comes from Selva Paranense, a humid evergreen subtropical forest characteristic of northeastern Argentina and adjoining Paraguay and Brasil.

Since almost $1.000 \mathrm{~km}$. of Dry Chaco, unsuitable for moisture loving ichneumonids, separate the Selva Paranense and the northwest Argentine humid forests, the present day range of Stangepelma in Argentina probably is disjunct. In this respect, Stange- 
pelma agrees with numerous other north Argentine humid forest insect genera, such as the ichneumonids Epirhyssa, Acrotaphus, Rhinium, Tretobasis, Megajoppa, Cryptojoppa, and Macrojoppa, the lepidopterous Morpho, and the pseudostigmatid zygopteron Mecistogaster, which enter Argentina only in the northwest and northeast, where they often are represented by closely allied or identical species. Evidently in the not distant past, perhaps during a Pleistocene glacial maximum, conditions were wetter than at present and humid forest biota ranged uniformly across the north of Argentina.

\section{ACKNOWLEDGEMENTS}

The holotype of $S$. argentinum was collected during fieldwork subsidized by the author's current U. S. National Science Foundation Grant (DEB 75-22426). I obtained the male paratype during a visit to the estate of Srs. Pablo, Arturo, and Adolfo Navajas Artaza at Establecimiento Las Marías near Governador Virasoro in Corrientes Province of Argentina. I am grateful for the hospitality of the Navajas Artaza, which made possible collecting in this unusually rich and little known area.

\section{REFERENCES}

GAuld, I. D.

1976. Classification of the Anomaloninae. Bull. British Mus. Natl. Hist. (Ent.) 33: 1-135.

TownEs, H. K.

1971. The Genera of Ichneumonidae, Part 4. Cremastinae to Diplazontinae. Mem. Amer. Ent. Inst. 17: 1-372. 

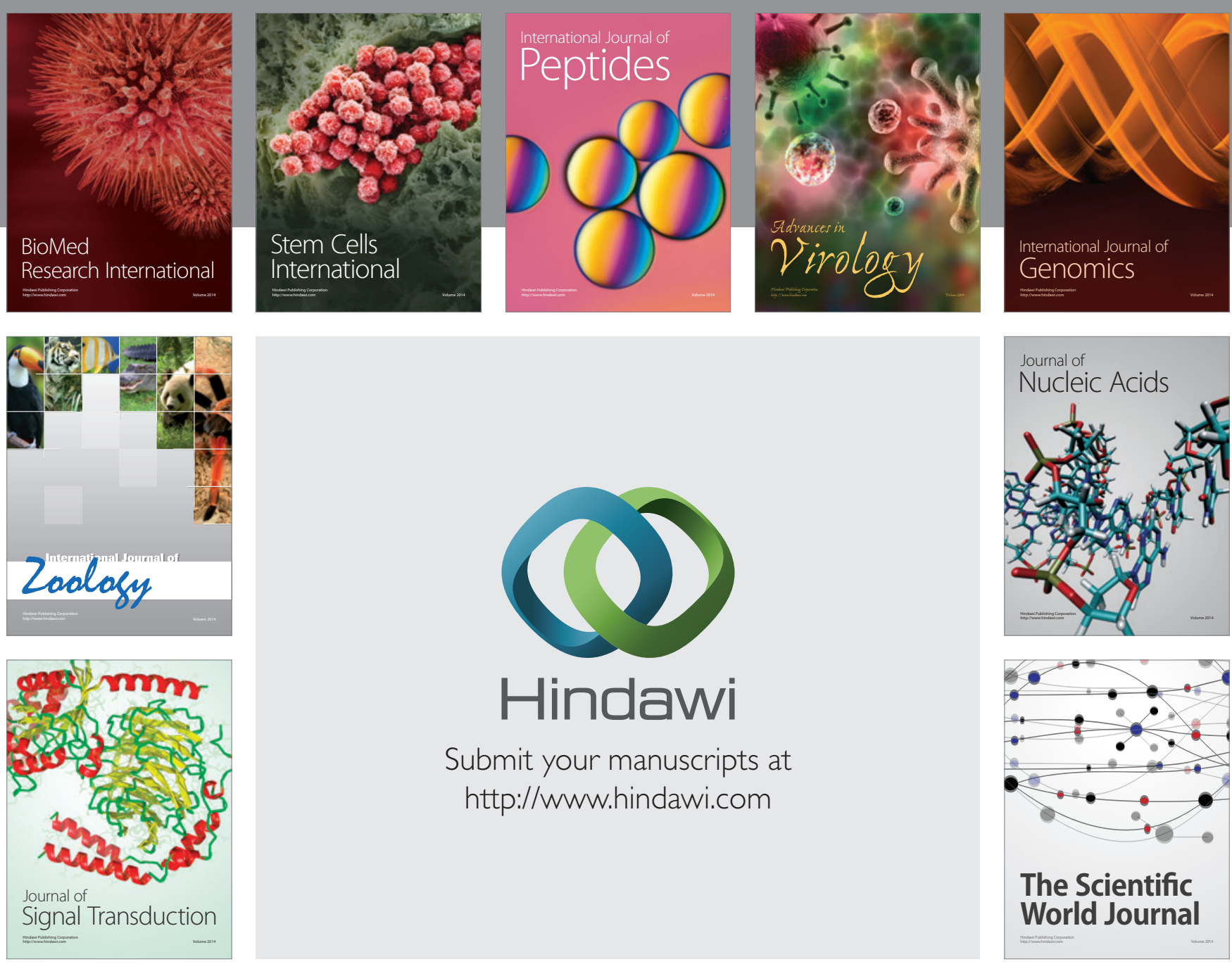

Submit your manuscripts at

http://www.hindawi.com
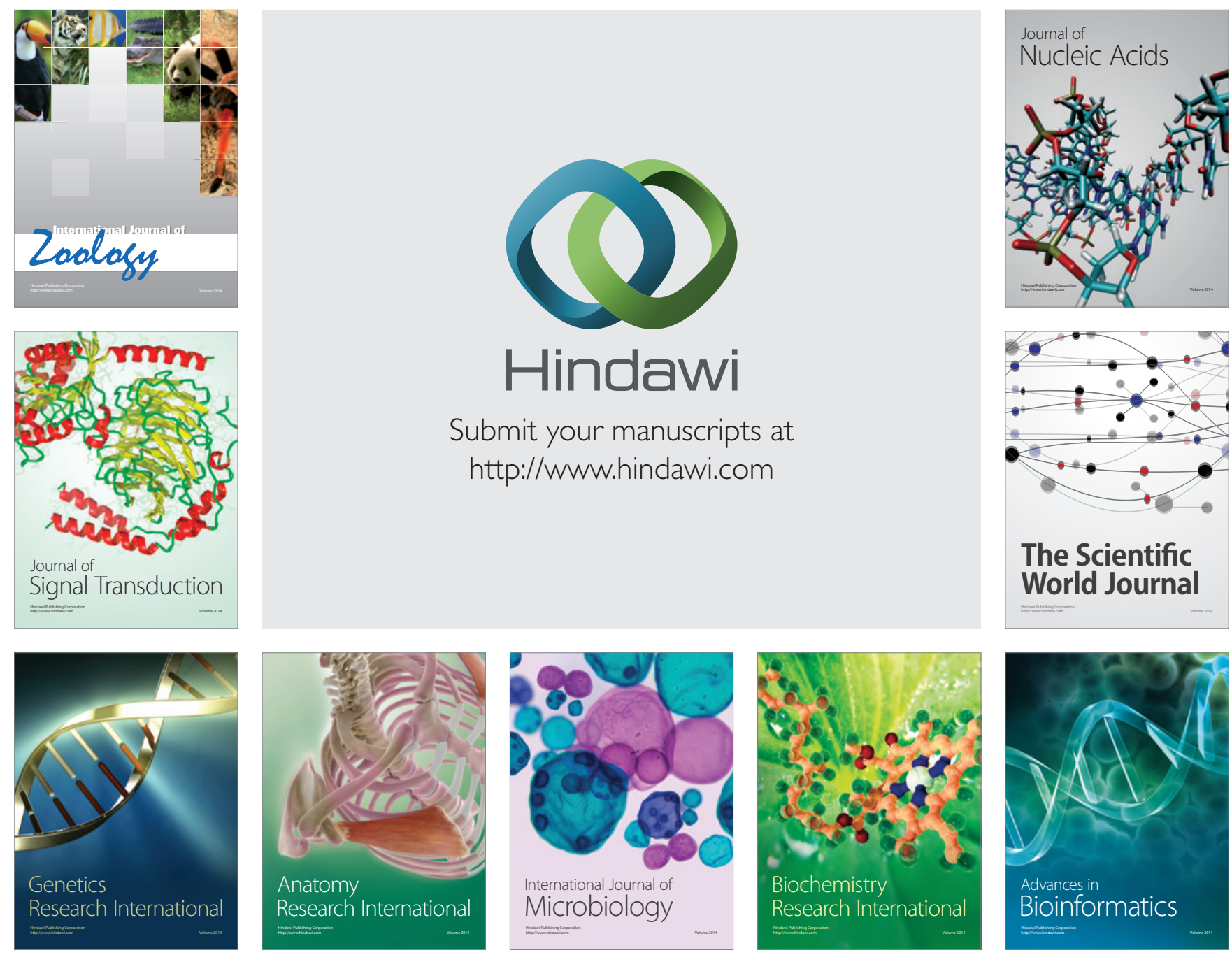

The Scientific World Journal
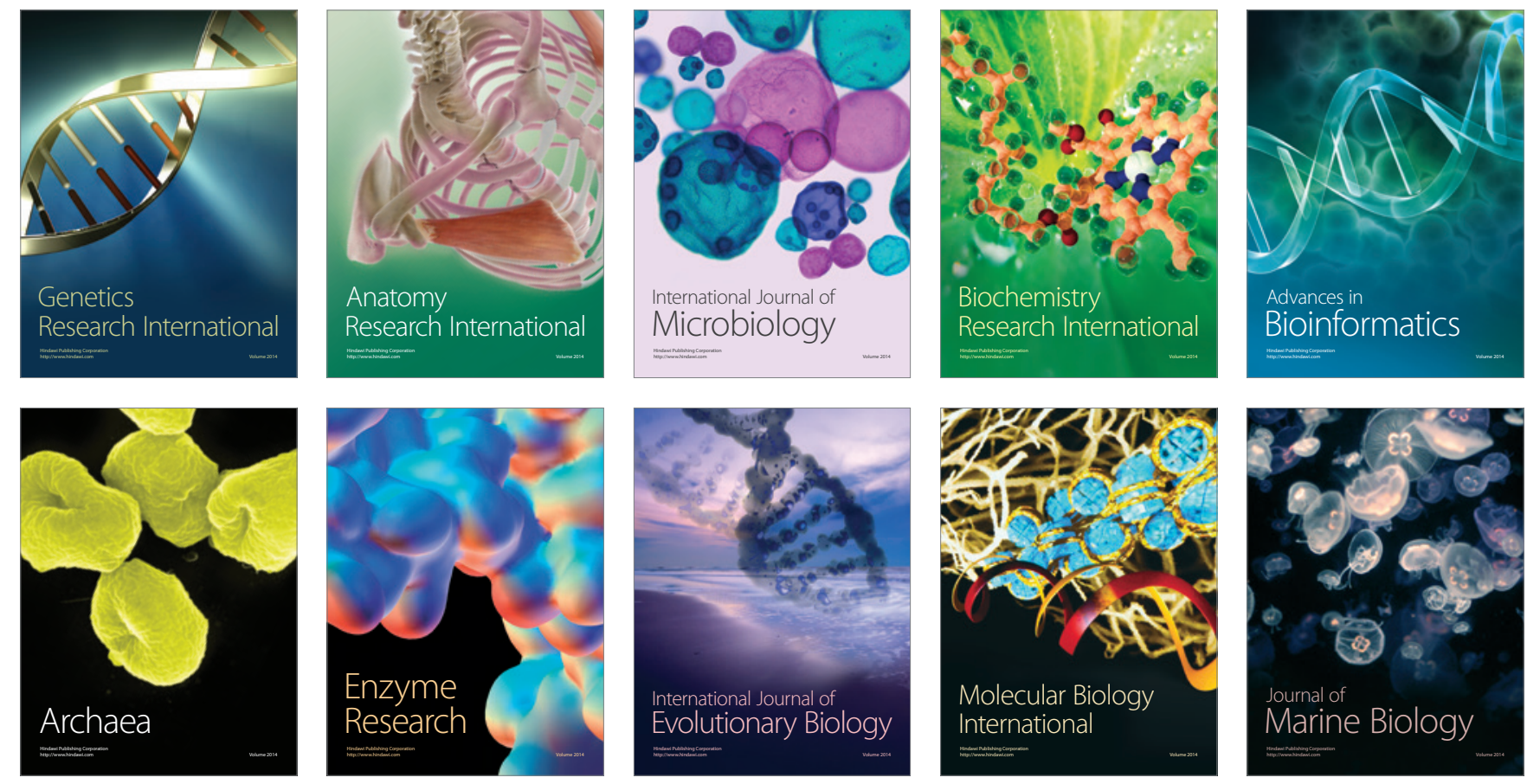